MM Research Preprints, 60-70

No. 17, Dec. 1998. Beijing

\title{
Automated Generation of Kempe Linkage and its Complexity
}

\author{
Xiao-Shan Gao and Chang-Cai Zhu \\ Institute of Systems Sciences, Academia Sinica \\ Beijing 100080, P.R. China
}

\begin{abstract}
It is a famous result of Kempe that we may design a linkage to generate any given plane algebraic curve. In this paper, Kempe's result is improved to give a precise algorithm for generating Kempe's linkage.. We also proved that for an algebraic plane curve of degree $n$, Kempe's linkage uses at most $O\left(n^{4}\right)$ links. We also report our efforts to implement a program which may generate the Kempe's linkage and simulate the generation process of the plane curves.
\end{abstract}

\section{Introduction}

Linkage design is the most basic and important kinds of mechanism design, the use of which can be traced back to the ancient age of human history. But, linkages became a hot research topic for mathematicians only in the nineteen century mainly due to Watt's invention of the steam engine, which needs a mechanism that can transform a cyclic movement into a movement along a straight line. The study of linkages attracts many great mathematicians of that time such as Cayley, Kempe, Hart, Darboux, Clifford, Darwin, Chebsheff, etc. In particular, Kempe proved the famous result that any algebraic curve can be drawn with a linkage.

There are basically two approaches of linkage design: exact design and approximate design. In the exact design approach, we design linkages to generate certain curves exactly. In the approximate design approach, we use simple linkages to generate certain curves approximately. Design of Kempe's linkage is a typical exact design problem. Approximate design is used more frequently in real mechanism design. Exact design is of more theoretical importance. It is used, e.g., in the complexity analysis of robotics [?]. Approximate design also has certain real applications, e.g., it is used in classrooms as assistant tools [?], or used in CAI (computer aided instruction) [?, ?]. Also, approximate design has potential industrial applications, e.g., certain fine $(* * *)$ machines may need linkage to generate exact curves.

In this paper, Kempe's result is improved to give a precise algorithm for generating Kempe's linkage.. We prove that for an algebraic plane curve of degree $n$, Kempe's linkage use at most $O\left(n^{4}\right)$ linkages. We also report our efforts to implement a program which may generate the Kempe's linkage and simulate the generation process of the plane curves. This program uses different approaches of computation to improve the efficiency. In this program, numerical optimization methods are used to simulate the generation process of the plane algebraic curves.

This paper is organized as follows. In Section 2, we will introduce some basic linkages. In Section 3, the design of Kempe's linkage and its complexity analysis are given. In Section 
4, we introduce the program to generate Kempe's linkage and to simulate the generation process.

\section{Some Basic Linkages}

\subsection{Straight Line Motion Linkage}

A basic question in mechanism is to design a linkage converting a cyclic motion into a line motion.

The most famous straight line motion linkage is the Peaucellier linkage, shown in Figure 1. In the diagram, $b=|P D|=|P B|, a=|A B|=|B C|=|C D|=|D A|$, and $|O P|=\mid$ $O A \mid=c$. Let $A C$ and $B D$ meet in point $E$. Thus

$$
P A \times P C=(P E-A E) \times(P E+A E)=P E^{2}-A E^{2}=b^{2}-D E^{2}-A E^{2}=b^{2}-a^{2}
$$

Therefore, point $C$ is the inversion point of point $A$ with respect to a circle whose center is point $O$. When point $A$ moves on a circle passing through point $O$, point $C$ 's trace is a line. Condition $|O P|=|O A|=c$ ensures that point $A$ will move on such a circle.

Because of the limited length of links, the locus of point $C$ is a line segment called Slot, shown as straight line $X Y$. This linkage is often used to constrain one endpoint to a line, when we say one point in a slot, it means that we use a Peaucellier linkage to do so.

Figure 2 is a modification of the Peaucellier linkage. Points $P, O$ are attached to a link $M N$ that is free to move in the plane. Thus, the slot itself can be moved freely. We say the slot is on a "platform".

\subsection{Translator and Rotator ( Distance Copier )}

Except straight line motion linkages we need other basic linkages. One linkage we need is a Translator. One translator easily thought is the linkage shown in Figure 3 , but of this linkage the distances between $A, B$ and $S, T$ are fixed, it isn't convenience. A better translator is shown in Figure 4 , of this translator, the distance between $A$ and $B$ doesn't exceed $2 a$ and the distance between $A$ and $S$ does not exceed $2 b$, that is to say, thee distance 
between $A$ and $B$ ( or $S$ ) can change. The translator is often used to translate one link to another place.

Another linkage is a Rotator which rotates one link with some angle. Figure 5 is such a linkage. This linkage uses many Peaucellier straight line motion linkages. It satisfies $|O A|=|O B|,|A C|=|B C|$, two "platforms" are attached to $O A$ and $O B$ such that $R$ is constrained to move in the slot along $O A$ and $S$ is constrained to move in the slot along $O B$, $I$ is constrained to move in the slot along $O C .|I R|=|I S|$, thus we have $|O R|=|O S|$, i.e., $O S$ is rotated to $O R$ by this rotator.

By combining a translator with a rotator, we obtain a distance copier(Figure 6) where the distance of $D T$ always equal the distance of $O R$.

\subsection{Angle Multiplicator and Adder}

First we can construct angle multiplicator, shown in Figure 7. The links satisfy $\frac{|O C|}{|O B|}=$ $\frac{|O B|}{|O A|}$. $O D A B$ and $O E B C$ are two isosceles trapezoids. Because $\triangle C O E \sim \triangle B O D, \angle C O E=$ $\angle B O D$, then $\angle C O B=\angle E O D$. Similarly $\triangle B O E \sim \triangle A O D$ implies $\angle B O A=\angle E O D$. Following the above, we have $\angle C O B=\angle B O A$. This is an angle multiplicator. Following this construction, we can get a $n$ times angle multiplicator. 
Now we construct an angle adder, shown in Figure 8. There are two angles $\angle A O B$ and $\angle A O C$. We want to get an angle $\angle A O D=\angle A O B+\angle A O C$. First we make the lengths of four links $O A, O B, O C, O D$ equal. Then we attach a "Distance Copier" to links $\mathrm{AB}$ and $\mathrm{CD}$. Thus $\angle C O D=\angle A O B$. The $\angle A O D$ is what we want, so we have constructed an angle adder.

\section{Generating Kempe Linkage}

In 1876, Kempe proved that any algebraic plane curve can be generated with a linkage [?]. In this section, we will show that for a given algebraic plane curve defined by the following equation

$$
f(x, y)=0
$$

how to construct a linkage that will generate the diagram of the above curve. Why we emphasis algebraic curves, first algebraic curves are the most basic and most important of all curves. Second, by Weierstrass Approximation Theorem, for any continuous function, there are a series of polynomial functions which uniformly approximate it. 


\subsection{Construction of the kempe Linkage}

Now suppose that a curve $f(x, y)=0$ is given. We construct a parallelogram (Figure 9) such that $\mathrm{OA}$ and $\mathrm{OB}$ have lengths $m$ and $n$ and form angles $\theta$ and $\varphi$ with the $\mathrm{X}$-axis respectively. $P$ is a point on the curve. Its coordinates are then given below.

$$
\begin{aligned}
& x=m \cos \theta+n \cos \varphi ; \\
& y=m \sin \theta+n \sin \varphi=m \cos (\pi / 2-\theta)+n \cos (\pi / 2-\varphi)
\end{aligned}
$$

Note that the products and powers of cosines can be expressed as the sum of cosines. If substituting equations (2) and (3) into (1), we shall have a sum of terms of the following form

$$
f(x, y)=\sum_{i=1}^{k} A_{i} \cdot \cos \left(a_{i} \varphi \pm b_{i} \theta \pm \beta_{i}\right)+C=0
$$

where $A_{i}$ and $C$ are constants; $a_{i}$ and $b_{i}$ are positive integers; and $\beta_{i}$ equals $\pi / 2$, or 0 . Note that if $a_{i}$ and $b_{i}$ are rational numbers, we can find a common denominator $d$ for them and treat $\varphi / d$ and $\theta / d$ as angle variables. We thus can always assume that $a_{i}$ and $b_{i}$ are integers.

For each term $A_{i} \cdot \cos \left(a_{i} \varphi \pm b_{i} \theta \pm \beta_{i}\right)$, we can use the angle multiplicator and adder to construct a link $O C_{i}$ such that $O C_{i}$ is of length $A_{i}$ and form an angle $a_{i} \varphi \pm b_{i} \theta \pm \beta_{i}$ with the X-axis. Using the Translators, we can construct a chain of links $O K_{1}, K_{1} K_{2}, K_{2} K_{3}, \ldots$, as shown in Figure 10, such that $K_{1}=C_{1}$ and $O C_{i} K_{i} K_{i-1}$ is a parallelogram. Therefore, point $K_{n}$ has x-coordinate :

$$
X=\sum_{i=1}^{n} A_{i} \cdot \cos \left(a_{i} \varphi \pm b_{i} \theta \pm \beta_{i}\right)=f(x, y)-C \text { (by equation (4) ). }
$$

If $P$ is moved along the given curve, its coordinates x, y satisfy: $f(x, y)=0$. Accordingly, the locus of the end point $K_{n}$ of the chain is

$$
X+C=0
$$

a straight line parallel to the $\mathrm{Y}$-axis. Conversely, if $K_{n}$ is moved along this line (with the help of a Peaucellier cell, for instance) point $P$ will generate the curve $f(x, y)=0$.Thus we finish the construction. 
Note that the real construction procedure is in the reverse order of the above construction steps, i.e., we will start from point $K_{n}$ on line $X+C=0$, construct points $K_{n-1}, \ldots, K_{1}$, and finally construct point $P$.

\subsection{The Complexity of the Kempe Linkage}

Now we discuss the complexity of the above constructive method, i.e., how many links are needed in the Kempe linkage?

Given an algebraic polynomial equation of degree $\mathrm{n} \sum_{0 \leq i+j \leq n} a_{i j} x^{i} y^{j}=0, a_{i j} \in R$,

Let $x=l \cos \theta+m \cos \varphi, y=l \sin \theta+m \sin \varphi$, then the above equation becomes

$0=\sum_{0 \leq i+j \leq n} a_{i j}(l \cos \theta+m \cos \varphi)^{i}(l \sin \theta+m \sin \varphi)^{j}$

$=\sum_{0 \leq i+j \leq n} a_{i j}\left(\sum_{s=0}^{i} c_{i}^{s} l^{s} m^{i-s} \cos ^{s} \theta \cos ^{i-s} \varphi\right)\left(\sum_{t=0}^{j} c_{j}^{t} l^{t} m^{j-t} \sin ^{t} \theta \sin ^{j-t} \varphi\right)$

$=\sum_{0 \leq i+j \leq n} a_{i j} \sum_{s=0}^{i} \sum_{t=0}^{j} c_{i}^{s} c_{j}^{t} j^{s+t} m^{i+j-s-t} \cos ^{s} \theta \cos ^{i-s} \varphi \sin ^{t} \theta \sin ^{j-t} \varphi$

Using the following formulas,

$$
\begin{aligned}
& \sin \theta=\cos \left(\theta-\frac{\pi}{2}\right) \\
& \cos ^{i} \theta=\frac{1}{2^{i-1}} \cos i \theta+c_{i-2} \cos (i-2) \theta+\cdots \\
& \cos \theta \cdot \cos \varphi=\frac{1}{2}[\cos (\theta+\varphi)+\cos (\theta-\varphi)]
\end{aligned}
$$

In the end the above equation can be translated into the following

$$
\sum_{0 \prec s+t \leq n} A_{s t} \cdot \cos \left(s \theta \pm t \varphi+\beta_{s t}\right)=-C
$$

The left items don't exceed $n^{2}+3 n$. To construct $s \theta \pm t \varphi$, we need to construct $\theta, 2 \theta$, $\cdots, n \theta, \varphi, 2 \varphi, \cdots, n \varphi$, analyzing the angle multiplicator,we only need the $n \theta$ and $n \varphi$ angle, these need $4(n+1)$ links; the angle adder needs fixed number links, assuming it as $N$, then it totally needs $\left(n^{2}+3 n\right) \cdot 2 N$ links; at last the translators need $1+3+5+\cdots+\left[2\left(n^{2}+3 n\right)-1\right]=$ $\left(n^{2}+3 n\right)^{2}$ links. Totally Kempe linkage need $4(n+1)+\left(n^{2}+3 n\right) \cdot 2 N+\left(n^{2}+3 n\right)^{2}$ links. So the complexity of Kempe constructive method is $0\left(n^{4}\right)$.

\section{Generating Kempe's Linkage with Geometry Expert}

In Section ??, we describe a method of constructing Kempe's linkage. According to this method, we can make a real linkage if a plane algebraic curve is given. But the best way is first to simulate the linkage on the computer. This section will report how to generate and simulate Kempe's linkage using software Geometry Expert [?].

\subsection{Automated Diagram Construction Software: Geometry Expert}

Automated geometry diagram construction (AGDC), also called engineering geometry, is to generate diagrams automatically if the geometric constraints in the diagram are given [?]. For instance, to draw a triangle if the lengths of its three medians are given. Three main approaches have been developed for automated diagram construction: the synthetic approach, the numerical approach, and the symbolic approach. [?]

Geometry Expert (GEX) [?] is a program for AGDC and automated geometry theorem proving and discovering. As an AGDC program, GEX can be used to build dynamic visual models which can be used to assist design of mechanisms. The following functions of GEX are used to generate Kempe's linkage. 
Geometric Construction. Basically speaking, GEX uses ruler and compass construction to draw geometry diagrams. Basic constructions include:

- Take free points.

- Take free points on a line or a circle.

- Take the intersection between lines and circles.

- Rotate a line segment.

- Ratio constructions.

Animation and Locus Generation. Through animation, the user may observe the generation process of curves or the figures of functions. In the case of Kempe's linkage, the plane curve is generated as the locus of some point during animation.

Equations Solving. To do animation, we need real time solutions of polynomial equation systems. If the diagram can be described in a constructive way, we can find the explicit solutions of the corresponding equations and the computation is very fast. In the general case, we need to solve a system of simultaneous equations. In GEX, a method based on the optimization techniques for solving geometric constraint problems is proposed. Experiments with this method show that it is fast and quite stable. Generally, a geometric constraint problem can be first translated into a system of equations:

$$
\begin{gathered}
f_{1}\left(x_{1}, \ldots, x_{n}\right)=0 \\
f_{2}\left(x_{1}, \ldots, x_{n}\right)=0 \\
\ldots \\
f_{m}\left(x_{1}, \ldots, x_{n}\right)=0 .
\end{gathered}
$$

Then the problem is how to solve this equation system. The optimization approach solves the equation system by converting it into finding $X$ at which the sum of squares

$$
\sigma(X)=\sum_{i=1}^{m} f_{i}(X)^{2}
$$

is minimal, where $X=\left(x_{1}, x_{2}, \ldots, x_{n}\right)$ is the set of variables. It is obvious that the equation system has a real solution $X^{*}$ if and only if $\min \sigma(X)$ is 0 . The problem of solving a system of equations is thus converted into the problem of finding the minimum of a real multi-variate function. The problem now can be solved by various well-developed numerical optimization methods [?, ?].

\subsection{Kempe's Linkage for Straight Lines}

To generate straight lines, we do not need Kempe's general construction method. We will design a special method which does no need to solve a system of simultaneous equations. Let the line be given by the following equation

$$
f(x, y)=a x+b y+c=0 .
$$

Let $x=n \cos \theta+n \cos \varphi, y=n \sin \theta+n \sin \varphi$. Substituting them into $f(x, y)=0$, we get a new equation

$$
f(x, y)=n \sqrt{a^{2}+b^{2}}(\cos (\theta \phi)+\cos (\varphi \phi))+c=0 .
$$


where $\phi$ is an angle satisfies $\cos (\phi)=\frac{a}{\sqrt{a^{2}+b^{2}}}, \sin (\phi)=\frac{b}{\sqrt{a^{2}+b^{2}}}$.

To generate the line $f(x, y)=0$, the sum of the two sides of the parallelogram $2 n$ must be greater than the distance from the original point to the line, i.e., $n$ must satisfy

$$
n>\frac{|c|}{2 \sqrt{a^{2}+b^{2}}} .
$$

The following is the construction procedure for the linkage

1. Construct a line $L M$ which is parallel with the y-axis and has a distance of $c$ to the $\mathrm{y}$-axis.

2. $F$ is a free point on line $L M$.

3. Construct a parallelogram $O G F H$ such that $O G=G F=F H=H O=n \sqrt{a^{2}+b^{2}}$.

4. Construction two lines $O J$ and $O I$ such that $\angle G O J=\phi, O J=n, \angle H O I=\phi, O J=n$.

5. Construct a parallelogram PJOI.

6. When point $F$ moves on line $L M$, point $P$ draws the line $f(x, y)=0$. Thus we have constructed a linkage drawing the line $f(x, y)=0$.

Example 4.1. We will construct a linkage generating the line defined by $f(x, y)=$ $x-y-2=0$.

Let $x=2 \cos \theta+\cos \varphi, y=2 \sin \theta+\sin \varphi$. Substituting them into $f(x, y)=0$, we get a new equation

$$
f(x, y)=2 \sqrt{2} \cos (\theta+\pi / 4)+\sqrt{2} \cos (\varphi+\pi / 4)-2=0 .
$$

Following the above construction procedure, we can construct a linkage, shown in figure 10. $A B C$ is a right isosceles triangle whose right angle edge $A B$ is of length $2 . L M$ is a line parallel to the y-axis and has a distance of 2 with the y-axis. $F$ is a free point on line $L M$. 
$O G F H$ is a parallelogram such that $O G=B C=2 \sqrt{2}, F G=\sqrt{2} . O G J$ and $O H I$ are right isosceles triangles. $P J O I$ is a parallelogram. When point $F$ moves on line $L M$, point $P$ draws the line $f(x, y)=0$. Thus we have constructed a linkage drawing the line $f(x, y)=0$.

Of course, the line $L M$ still need to be generated by a Peaucellier linkage. The device designed here is actually to transform one line to another line.

\subsection{Kempe's Linkage for Non-linear Curves}

To generate a non-linear curve $f(x, y)=0$, we need the following steps.

1. Construct a parallelogram $O A P B$ with sides $n$ and $m$. Let $\theta$ and $\varphi$ be the angles from $O A, O B$ to the $\mathrm{x}$-axis respectively.

2. To construct the linkage, we will not exactly follow the construction procedure described in Section ??. Since the construction here is just a simulation, we will use the simplest method to compute the linkage. For instance, to construction a link $O C_{i}$ such that $C_{i}$ has x-coordinate $A_{i} \cos \left(a_{i} \theta+b_{i} \varphi+\phi_{i}\right)$, we only need the following constructions:

- Take points $O$ and $X$ such that $O$ is the original point and $O X$ is the x-axis.

- Rotate $O A$ to $O A^{\prime}$ such that $O A^{\prime}$ forms angle $a_{i} \theta$ with the x-axis.

- Rotate $O B$ to $O B^{\prime}$ such that $O A^{\prime}$ forms angle $a_{i} \varphi$ with the x-axis.

- Rotate $O B^{\prime}$ to $O K$ such that $\angle K O B^{6}=\angle A O X$.

- Take a point $C_{i}$ on $O K$ such that $\left|O C_{i}\right|=A_{i}$.

In GEX, all the above constructions will be realized with numerical computation.

At the final step, we will construct points $K_{1}, \ldots, K_{n}$ and a line $l: x+C=0$. If point $K_{n}$ moves on line $l$, point $P$ will generate the plane curve.

3. We will use a Peaucellier linkage to move point $K_{n}$ on line $l$, and for each position of $K_{n}$ using the optimization method to compute positions of all other points. Finally, if we remember the positions for points $P$. These points will form the desired plane curve.

In order to program the above procedure, we need to answer two questions.

First, we need to determine the value of $l$ and $m$. If the values of $l$ and $m$ are too small, the linkage may not reach the area of the curve. For convenience, let us assume that $m=n$. Then $x=n(\cos \theta+\cos \varphi), y=n(\sin \theta+\sin \varphi)$. Generally speaking, to reach the curve $f(x, y)=0$, the following equation system must have solutions.

$$
\left\{\begin{array}{l}
f(x, y)=0 \\
x^{2}+y^{2}=(2 n)^{2}
\end{array}\right.
$$

Let $N$ be the minimal value of $n$ such that the above system has solutions. So the $n$ must satisfy $n>N$.

Second, for a given $n$ we need to determine the starting and ending positions for point $K_{n}$. To find these positions, we first find the solutions of the equation system (5). Let $P_{1}, \ldots, P_{d}$ be the solutions. For each $P_{i}$, we substitute its value (for point $P$ ) into the equation system of the linkages constructed above and find the positions for point $K_{n}$. Let $y_{1}$ and $y_{2}$ be 
the minimal and the maxima y-coordinates of all the possible solutions for point $K_{n}$. Then $\left(-C, x_{1}\right)$ and $\left(-C, x_{2}\right)$ are the starting and ending points for $K_{n}$.

Example 4.2. We will construct a linkage to generate $f(x, y)=x^{2}-y=0$. Let $x=n \cos \theta+n \cos \varphi, y=n \sin \theta+n \sin \varphi$. Substitute then into $f(x, y)=0$, we have

$f(x, y)=n\left(\cos (\theta+\pi / 2)+\cos (\varphi+\pi / 2)+\frac{n}{2} \cos (2 \theta)+\frac{n}{2} \cos (2 \varphi)+n \cos (\theta+\varphi)+n \cos (\theta-\varphi)\right)+n^{2}=0$.

Therefore, the straight line $l$ is

$$
X+n^{2}=0
$$

Here is how to construct this linkage.

1. Take two points $O$ and $C$ such that $n=|O C|$ and $O C$ is the x-axis.

2. Take two free points $A$ and $B$ on the circle with center $O$ and radius $n$ and construct a parallelogram $O A P B$.

3. Rotate $O A$ to $O H$ such that $\angle H O A=\pi / 2$. Rotate $O B$ to $O I$ such that $\angle I O B=\pi / 2$. Points $H$ and $I$ will give terms $n \cos (\theta+\pi / 2)$ and $n \cos (\varphi+\pi / 2)$.

4. Rotate $O A$ to $O J$ such that $\angle J O A=\angle A O C$. Take a point $J_{1}$ on $O J$ such that $O J_{1}=O C^{2} / 2$. Point $J_{1}$ will give term $\frac{n^{2}}{2} \cos (2 \theta)$.

5. Rotate $O B$ to $O K$ such that $\angle K O B=\angle B O C$. Take a point $K_{1}$ on $O K$ such that $O K_{1}=O C^{2} / 2$. Point $K_{1}$ will give term $\frac{n^{2}}{2} \cos (2 \varphi)$.

6. Rotate $O B$ to $O L$ and $O M$ such that $\angle L O B=\angle A O C$ and $\angle M O B=\angle C O A$. Take points $L_{1}$ and $M_{1}$ on $O L$ and $O M$ such that $O L_{1}=O M_{1}=O C^{2}$. Point $L_{1}$ and $M_{1}$ will give terms $n^{2} \cos (\theta+\varphi)$ and $n^{2} \cos (\theta-\varphi)$.

7. Construct five parallelograms to get the final point $S$. If $S$ moves on line $X+n^{2}=0$. Point $P$ will generate curve $y=x^{2}$.

To determine the starting and ending points, we need to solve the equations:

$$
y-x^{2}=0, x^{2}+y^{2}-4 n^{2}=0 .
$$


Two solutions of the above equations are $\pm \sqrt{\frac{\sqrt{1+16 n^{2}}-1}{2}}$. Then the two end points for point $P$ are

$$
p_{1}=\left(-\sqrt{\frac{\sqrt{1+16 n^{2}}-1}{2}}, \frac{\sqrt{1+16 n^{2}}-1}{2}\right), p_{2}=\left(\sqrt{\frac{\sqrt{1+16 n^{2}}-1}{2}}, \frac{\sqrt{1+16 n^{2}}-1}{2}\right) .
$$

The corresponding positions for point $S$ can be approximately found by moving point $S$ to the points $p_{1}$ and $p_{2}$ and observing the positions of $S$.

\section{References}

[1] K.H. Elster (ed), Modern Mathematical Methods of Optimization, Akademie Verlag, 1993.

[2] X. S. Gao, Automated Geometry Diagram Construction and Engineering Geometry, this volume.

[3] X. S. Gao, J. Z. Zhang, and S. C. Chou, Geometry Expert, Nine Chapter Pub., 1998 (in Chinese).

[4] X. S. Gao, C. C. Zhu, and Y. Huang, Building Dynamic Mathematical Models with Geometry Expert, II. Linkages, Proceedings of the Third Asian Symposium on Computer Mathematics, ed. Z. B. Li, pp. 15-22, LanZhou Univ. Press, 1998.

[5] J. Hopcroft, D. Joseph and A. S. Whitesides, Movement Problems for 2-Dimensional Linkages, Siam J. Comp., 13(3), 610-629.

[6] M. Isoda, Developing the Curriculum for Curves Using History and Technology, Proceedings of the Third Asian Technology Conference in Mathematics, eds W.C. Yang, pp. 82-89, Springer, 1998.

[7] N. Jakiw, Geometer's Sketchpad, User guide and Reference Manual, Key Curriculum Press, 1994.

[8] A. S. Hall, Kinematics and Linkage Design, Prentice-Hall, INC, 1962, Englewood, Cliffs.

[9] R. S. Hartenberg and J. Denavit, Kinematic Synthesis of Linkages McGraw-Hill Book, 1964.

[10] J. A. Hrones and G. L. Nelson, Analysis of the Four-bar Linkage, MIT Press and Wiley, New York, 1951.

[11] A. B. Kempe, On a general method of describing plane curves of the nth degree by linkwork, Proc. of L.M.S., p.213-216, 1876; voir aussi Messenger of Math., T. VI., p.143-144.

[12] F. V. Morley, American Mathematical Monthly 31 pp.71-77 (1924)

[13] G.L. Nemhauser, A.H.G. Rinnooy Kan and M.J. Todd (eds), Optimization, Elsevier Science Publishers B.V., 1989.

[14] Wu Wen-tsün, Basic Principles of Mechanical Theorem Proving in Geometries, Springer-Verlag, 1993.

[15] R. C. Yates, Geometrical Tools, A Mathematical Sketch and Model Book, Educational Pub. Inc., Saint Louis, 1949. 